\title{
CALR Exon 9 Mutant Peptide Vaccine/Montanide ISA-51
}

National Cancer Institute

\section{Source}

National Cancer Institute. CALR Exon 9 Mutant Peptide Vaccine/Montanide ISA-51. NCI

Thesaurus. Code C160197.

A peptide vaccine consisting of a calreticulin (CALR) mutant peptide, CALRLong 36, and montanide ISA 51 with potential antineoplastic activity. Upon vaccination, the CALR exon 9 mutant peptide vaccine may stimulate the host immune system to mount a cytotoxic T-lymphocyte (CTL)-mediated response against tumor cells harboring mutations in exon 9 of the calreticulin gene. CALR, an endoplasmic reticulum chaperone protein that normally facilitates protein folding, immune response, and hematopoiesis, is often mutated in patients with chronic myeloproliferative neoplasms. In its mutant form, CALR is secreted into the plasma, where it binds to and activates the thrombopoietin receptor MPL and initiates downstream JAK/STAT signaling. Montanide ISA-51, also known as incomplete Freund's adjuvant or IFA, is a stabilized water-in-oil (w/o) emulsion adjuvant containing mineral oil with mannide oleate added as a surfactant that non-specifically stimulates cell-mediated immune responses to antigens. 